The Last Word

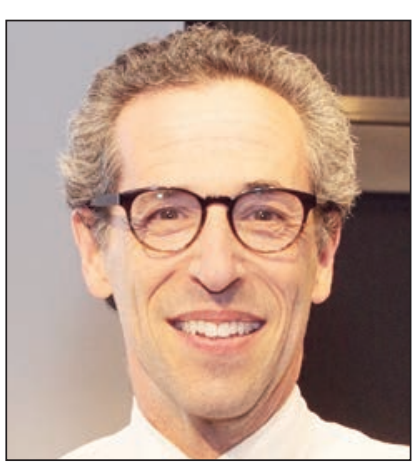

Andrew D. Leavitt, MD

Andrew D. Leavitt, MD, is Professor of Medicine and Laboratory Medicine and Director of Nonmalignant Hematology, Division of Hematology/Oncology at the University of California, San Francisco. He studied medicine at Harvard Medical School and did his residency in internal medicine at the University of Michigan, Ann Arbor and fellowship in Hematology at the University of California, San Francisco.

\section{A Small Step Closer But Not Yet There: Oral Anti-Xa Agents and the Treatment of Cancer- Associated Venous Thromboembolism}

\author{
Andrew D. Leavitt, MD
}

Patients with cancer have upwards of a 7-fold increased risk of venous thromboembolism (VTE; deep vein thrombosis and pulmonary embolism) and a 2-to 3-fold increased risk of VTE recurrence compared with patients without cancer. ${ }^{1}$ VTE risk varies across cancer types, with pancreatic, brain, lung, and ovarian cancers associated with some of the highest risk. ${ }^{1}$ In addition to symptomatic VTE, patients with cancer are often diagnosed with apparently asymptomatic, often called incidental, VTE found on staging or restaging scans.

VTE treatment decisions and ongoing management can be difficult in patients with cancer due to comorbidities, drug interactions during concomitant chemotherapy, and limited tolerance for oral medications. The CLOT trial reported in $2003 \mathrm{did}$ much to establish parenteral anticoagulation as the preferred approach over oral anticoagulation for treating VTE in patients with cancer. ${ }^{2}$ This approach is now recommended in various professional society guidelines, including those of ASCO. ${ }^{3}$ The CLOT trial demonstrated that parenteral anticoagulation is associated with reduced VTE recurrence without an increased bleeding risk when compared with oral anticoagulants.

At the time, oral anticoagulant options were limited to vitamin $\mathrm{K}$ antagonists, such as warfarin. The upside of improved outcomes in terms of reduced VTE recurrence brought the downside of pain, difficulty, and inconvenience of daily injections. Moreover, although the CLOT trial involved 1 daily subcutaneous injection of dalteparin, many patients in clinical practice receive parenteral anticoagulants that require twice-daily subcutaneous injections. Not all patients are able or willing to administer parenteral agents once or twice per day over extended periods, which often creates difficult clinical decisions for patients and providers. Therefore, although the CLOT trial provided evidence for improved VTE recurrence outcomes with parenteral anticoagulants, implementing the findings remains a challenge for patients and providers.

With the FDA approval of oral anti-Xa agents in late 2012 (rivaroxaban) and in 2014 (apixaban) for VTE treatment and recurrence prevention, patients and providers gained access to the first new oral anticoagulant treatment options in $>60$ years. The drugs were approved after being shown to be at least as effective and as safe as warfarin, and in some situations more effective and safer. ${ }^{4,5}$

Providers who care for patients with cancer were excited by the new options, but disappointed that the initial trials did not directly address efficacy and safety in this patient population. Some providers found solace and support in post hoc analyses that suggested similar efficacy and safety for the new drugs in patients with cancer as for the total study cohort. However, many providers have stayed on the sidelines, avoiding use of the new drugs until there are properly designed studies showing efficacy and safety in patients with cancer. Moreover, the original studies compared anti-Xa agents with oral vitamin $\mathrm{K}$ antagonists, and therefore the data do not directly address the question of efficacy and safety as they relate to parenteral anticoagulation, which is often considered the gold standard for treatment of VTE in patients with cancer.

In this landscape of limited data and many important unanswered questions, we welcome the report by Kohn et al, titled "Effectiveness and Safety of Rivaroxaban in Patients With Cancer-Associated Venous Thrombosis," elsewhere in this issue. Using US Truven Health Analytics MarketScan Databases, which combine commercial and Medicare supplemental databases, the authors performed a retrospective, single-arm analysis of results for patients with cancer-associated venous thrombosis treated with rivaroxaban as outpatients.

Using health insurance claims from November 2012 (when rivaroxaban received FDA approval) through September 2015, the authors identified all patients aged $\geq 18$ years 
with active cancer who also had $\geq 1$ hospitalization or emergency department discharge diagnosis coded for VTE (index event) and who received rivaroxaban as their first outpatient anticoagulant within 30 days of the index event. Moreover, patients needed to have at least 180 days of continuous medical/prescription benefits before the index VTE, and patients with a prior claim for VTE, atrial fibrillation, valve disease, or those receiving any anticoagulation during the baseline period were excluded. Primary end points were recurrent VTE, major bleed, and all-cause mortality or a claim for hospice care within 180 days after the index event. Because only in-hospital deaths are captured by their screening system, they combined in-hospital death with need for hospice care as a single end point, as has been done by others. ${ }^{7}$ An intent-to-treat approach was used.

The authors identified 949 patients with an active cancer diagnosis started on rivaroxaban within 30 days of the index VTE over the roughly 3-year study period. The time from active cancer diagnosis to index VTE event was $\leq 90$ days for $27 \%$ of patients, 91 to 180 days for $19 \%$ of patients, and $>180$ days for $54 \%$ of patients. Deep vein thrombosis only was reported in $56.4 \%$ of patients, and $43.6 \%$ had pulmonary embolism with or without deep vein thrombosis. The authors report full-cohort cumulative incidence estimates for recurrent VTE (4.0\%; 95\% CI, 2.8\%-5.4\%), major bleeding (2.7\%; 95\% CI, 1.7\%$4.0 \%)$, and mortality/hospice care (11.3\%; 95\% CI, 9.2\%-13.6\%). Cancer diagnoses included breast $(25.5 \%)$, genitourinary including prostate $(21 \%)$, gastrointestinal $(17.3 \%)$, hematologic $(13.2 \%)$, lung $(11.5 \%)$, gynecologic $(7.4 \%)$, pancreatic $(5.8 \%)$, and other $(39.5 \%)$, and $42.6 \%$ of patients had metastatic disease.

As we await prospective randomized trial data focusing on patients with cancer who have VTE, reports such as the one by Kohn et al provide some initial insights for providers. At first blush, it suggests a reasonable safety profile when compared with historical recurrence and bleeding risks for other anticoagulants. However, on closer inspection, the applicability of the findings is unclear due to a strong risk of patient selection bias. Such studies can also fall victim to diagnosis coding errors, and one must wonder about the general applicability given that PPO patients represent $55.6 \%$ of the patient population but $\mathrm{HMO}$ patients only $9.3 \%$. Additional study weaknesses include the lack of information about brain metastasis, the lack of clinical variables that influence patient and provider treatment decisions, and the lack of a control arm. Although the research used accepted standards and approaches, given these limitations, the report provides only a modest advance in the field of cancer-associated thrombosis management, and in particular, the use of rivaroxaban for such patients.

Prospective trials of direct oral anticoagulants are underway with various anti-Xa agents in patients with cancer, and the results are starting to reach the public domain. This is a welcome milestone for patients and providers. Edoxaban was FDA-approved in 2015 , and investigators recently reported a prospective, randomized clinical trial comparing edoxaban and dalteparin in the treatment of cancer-associated VTE. ${ }^{8}$

Wisely, dalteparin was dosed similarly to the CLOT trial. ${ }^{2,8}$ Edoxaban was found to be noninferior to subcutaneous dalteparin when using the composite outcome of recurrent VTE and major bleeding. However, the study raised real concerns for bleeding complications in some patient subsets, most notably those with gastrointestinal cancers, a finding that highlights the importance of prospective randomized studies. Initial data from the randomized prospective SELECT-D trial comparing rivaroxaban with dalteparin was presented in December 2017 at the American Society of Hematology annual meeting. ${ }^{9}$ However, findings related to safety, efficacy, and specific cancer types will only become clear after the trial is complete and made available in a peer-reviewed journal.

It is exciting to be on the cusp of new and emerging data that will help advance the care of patients with cancer-associated venous thrombosis, but we need much more data to allow us to provide reasoned and substantiated guidance. We need prospective randomized studies that provide detailed information on different cancer types, presence or absence of brain metastases, results during ongoing chemotherapy versus after treatment cycles have ended, and other critical clinical variables that come into play when making treatment decisions with patients. The field also needs studies that specifically address the question of treatment duration, treatment value for apparently asymptomatic VTE identified on staging or restaging scans, and clinical value of low-dose extension treatment. We owe it to our patients. 
Leavitt

\section{References}

1. Timp JF, Braekkan SK, Versteeg HH, et al. Epidemiology of cancer-associated venous thrombosis. Blood 2013;122:1712-1723.

2. Lee AY, Levine MN, Baker RI, et al. Low-molecular-weight heparin versus a coumarin for the prevention of recurrent venous thromboembolism in patients with cancer. N Engl J Med 2003;349:146-153.

3. Lyman GH, Khorana AA, Kuderer NM, et al. Venous thromboembolism prophylaxis and treatment in patients with cancer: American Society of Clinical Oncology clinical practice guideline update. J Clin Oncol 2013;31:2189-2204.

4. Bauersachs R, Berkowitz, SD, Brenner B, et al. Oral rivaroxaban for symptomatic venous thromboembolism. N Engl J Med 2010;363:2499-2510.

5. Agnelli G, Buller HR, Cohen A, et al. Oral apixaban for the treatment of acute venous thromboembolism. N Engl J Med 2013;369:799-808.

6. Agnelli G, Buller HR, Cohen A, et al. Oral apixaban for the treatment of venous thromboembolism in cancer patients: results from the AMPLIFY trial. J Thromb Haemost 2015;13:2187-2191.

7. Ooba N, Setoguchi S, Ando T, et al. Claims-based definition of death in Japanese claims database: validity and implications. PLoS One 2013;8;e66116.

8. Raskob GE, van Es N, Verhamme P, et al. Edoxaban for the treatment of cancer- associated venous thromboembolism. N Engl J Med 2018;378:615-624.

9. Young A, Marshall A, Thirlwall J, et al. Anticoagulation therapy in selected cancer patients at risk of recurrence of venous thromboembolism: results of the SELECT-D pilot trial [abstract]. Blood 2017;130:Abstract 625. 